(c) 2017 IEEE. Personal use of this material is permitted. Permission from IEEE must be obtained for all other uses, in any current or future media, including reprinting/republishing this material for advertising or promotional purposes, creating new collective works, for resale or redistribution to servers or lists, or reuse of any copyrighted component of this work in other works.

DOI: 10.1109/LAWP.2016.2631889 


\title{
Assessment of a Millimeter-Wave Antenna System for MIMO Radar Applications
}

\author{
Claudia Vasanelli, Student Member, IEEE, Rahul Batra, Adolfo Di Serio, Frank Boegelsack, \\ and Christian Waldschmidt, Senior Member, IEEE
}

\begin{abstract}
Multiple-Input Multiple-Output (MIMO) radars show improved angular resolution compared to traditional phased-array radar systems. Exploiting the advantages of MIMO radars could thus significantly improve the performances of the next generation of automotive radar sensors. The placement and the characteristics of the antenna arrays affect the performance of the MIMO system. For such applications, different metrics like the ambiguity function of the antenna system must be accurately evaluated. This paper presents a detailed characterization of a MIMO antenna configuration at $\mathrm{mm}$-wave frequencies. To prove the effectiveness of this method, a five-element non-uniform antenna configuration based on cavity antenna arrays has been designed and tested.
\end{abstract}

Index Terms-Antenna measurements, MIMO radar, millimeter-wave antennas, automotive applications

\section{INTRODUCTION}

Thanks to the significant progress in mm-wave technology, radar sensors are nowadays key components of driving assistance systems. Adaptive cruise control and emergency braking are few examples of the features that exploit mm-wave radars and that can be found both in luxury vehicles and family cars.

Automotive radars can accurately measure distance and velocity of the surroundings targets and are robust against bad weather conditions [1]. They operate in Europe in the frequency range from $76 \mathrm{GHz}$ to $81 \mathrm{GHz}$.

An increase in the reliability of driving assistance systems could pave the way for autonomous driving. To achieve this goal, the next generation of radar sensors must definitely improve the capability of perception of the environment [2]. This means for example to improve the angular resolution in a multi-target scenario [3]. The well-known Rayleigh criterion [1] relates the angular resolution with the dimension of the sensing element, i.e. the antenna array. Larger antenna systems allow to improve the angular resolution of the sensor. However, increasing the number of elements in the array raises the overall dimension of the sensor and the complexity of the system. This solution is thus not feasible in any practical case.

An alternative solution is to exploit the advantages of MIMO radars. Unlike phased-array systems, a MIMO radar transmits multiple probing signals [4] that enable the synthesis of a virtual aperture wider than the physical one. The larger virtual aperture provides then a better angular resolution.

The properties of the virtual array are not only affected by the waveforms, but also by the physical placement of the transmitting and receiving elements. Moreover, the antenna position and characteristic affect several system metrics, like

The authors are with the Institute of Microwave Engineering, Ulm University, 89081 Ulm, Germany (e-mail: claudia.vasanelli@uni-ulm.de) the field of view (FoV) of the sensor and the achievable unambiguous range in the direction of arrival (DoA) estimation. Although much theoretical work has been published on the antenna placement strategy for MIMO radars, for example [5], to the best of authors' knowledge the characterization and the assessment of antenna configurations for MIMO radars has not yet been deeply analyzed. Indeed, in this case the classical antenna evaluation criteria, like the radiation pattern in the two main cuts, are not sufficient for the complete characterization of the antenna system.

In this work a detailed characterization of a non-uniform array configuration for a future MIMO radar sensor is carried out. The expected and simulated resolution properties of the antenna system are verified by means of measurement results.

\section{Signal Model}

Since the waveform optimization falls outside the scope of this paper, it is assumed that independent orthogonal signals are transmitted.

The system is composed of $M_{t}$ transmitting and $M_{r}$ receiving antenna arrays placed on a one-dimensional lattice, as can be seen in Fig. 1. Only a multistatic scenario will be investigated, i.e. the transmitting and the receiving antenna arrays are at different positions. To derive the virtual array definition and to describe the properties of the system, the targets are assumed in the far-field of the antenna array. Due to this assumption, plane waves impinge on the targets and the directions of propagation of the incoming signals (the angle $\vartheta$ drawn in Fig. 1) are the same for each element of the array. Finally, in this paper only the azimuth plane is of interest.

According to the reference system described in Fig. 1, the first antenna array is placed in the origin. Let $x_{T k}$ and $x_{R l}$ denote the position of the transmitters and receivers respectively with $k=1, \ldots, M_{t}$ and $l=1, \ldots, M_{r}$. Then, in the ideal case of isotropic radiators the transmitter and receiver steering vectors associated to the direction $\vartheta$ are defined as

$$
\boldsymbol{a}(\vartheta)=\left[\begin{array}{c}
\mathrm{e}^{\mathrm{j} 2 \pi \frac{x_{T 1}}{\lambda_{0}} \sin (\vartheta)} \\
\vdots \\
\mathrm{e}^{\mathrm{j} 2 \pi \frac{x}{\lambda_{0} M_{t}}} \sin (\vartheta)
\end{array}\right], \boldsymbol{b}(\vartheta)=\left[\begin{array}{c}
\mathrm{e}^{\mathrm{j} 2 \pi \frac{x R 1}{\lambda_{0}} \sin (\vartheta)} \\
\vdots \\
\mathrm{e}^{\mathrm{j} 2 \pi \frac{x_{R} M_{r}}{\lambda_{0}} \sin (\vartheta)}
\end{array}\right]
$$

where $\lambda_{0}$ is the wavelength in free space.

The virtual array steering vector associated to the MIMO antenna configuration can be hence written as

$$
\boldsymbol{y}(\vartheta)=\boldsymbol{a}(\vartheta) \otimes \boldsymbol{b}(\vartheta),
$$

where $\otimes$ refers to the Kronecker product. Thus, given $M_{t}$ transmitters and $M_{r}$ receivers, we can derive a virtual array with $M_{t} M_{r}$ number of elements. 


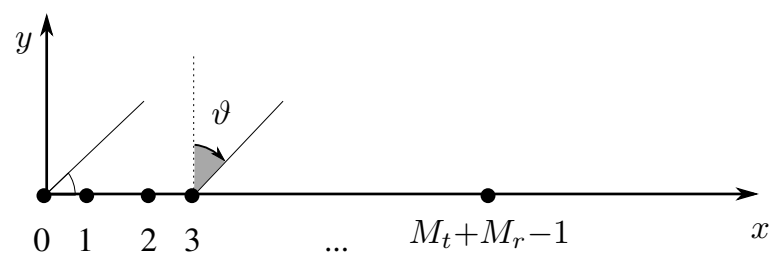

Fig. 1. Arrangement of the arrays and description of the reference system.

It is straightforward then to introduce the ambiguity function $(\mathrm{AF})$, that is a significant metric to evaluate the performances of the antenna system in DoA estimation problems. According to [6] and [7], the AF of the virtual array is defined as

$$
\chi\left(\vartheta_{i}, \vartheta_{j}\right)=\frac{\left|\boldsymbol{y}\left(\vartheta_{i}\right)^{H} \boldsymbol{y}\left(\vartheta_{j}\right)\right|}{\left\|\boldsymbol{y}\left(\vartheta_{i}\right)\right\|\left\|\boldsymbol{y}\left(\vartheta_{j}\right)\right\|},
$$

where the symbol $(\cdot)^{H}$ denotes the complex conjugate (Hermitian) vector. To take into account the radiation property of the antennas too, the steering vector must be multiplied by the complex antenna radiation pattern $C(\vartheta)$ [8].

The AF shows the ambiguity and resolution characteristics of the virtual array. Both are fundamentally related to the geometry of the virtual array [6], which then in turn depends on the placement of the real transmitting and receiving antennas.

If identical antennas are employed, or identical sets of transmitters and receivers are used, all the transmitter-receiver pairs that compose the virtual array are identical, too. By simple calculations, it is possible to prove that in these special cases the properties of the $\mathrm{AF}$ are the same as for the isotropic radiators. The resolution and ambiguity properties of the antenna system are thus affected only by the geometrical displacement of the arrays and not by the complex radiation pattern $C(\vartheta)$. Indeed, it can be proved that when the elements of the virtual array are identical the numerator of (2) can be written as

$$
\left|\boldsymbol{y}\left(\vartheta_{i}\right)^{H} \boldsymbol{y}\left(\vartheta_{j}\right)\right| \propto\left|A_{C(\vartheta)}\right| \chi^{\prime}\left(\vartheta_{i}, \vartheta_{j}\right),
$$

where the first term $\left|A_{C(\vartheta)}\right|$ depends on the amplitudes of the complex radiation patterns and $\chi^{\prime}\left(\vartheta_{i}, \vartheta_{j}\right)$ is the value that would be calculated in the isotropic radiator case. The first term can be then normalized by the denominator in (2).

In any practical case every transmitter-receiver pair is different from the others, therefore their complex radiation pattern must be included in (2).

\section{Antenna CONFiguration}

\section{A. Optimal Placement of the Antennas}

A genetic algorithm is employed for finding the optimal antenna placement [9]. The algorithm starts with determining the virtual array length using the Rayleigh criterion for a desired angular resolution as [1]

$$
\Delta \varphi=1.22 \frac{\lambda_{0}}{d_{v}}
$$

where $\Delta \varphi$ is the desired angular resolution and $d_{v}$ is the total virtual array aperture. It is important to notice that the Rayleigh criterion sets only a theoretical value on the achievable angular resolution and it takes into account only

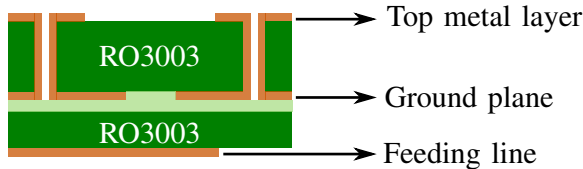

Fig. 2. Stackup of the proposed antenna array.

the aperture size. However, the virtual aperture that can be synthesized by the MIMO radar is in general composed of a non-uniform displacement of virtual array elements and thus the actual angular resolution can be different from the value predicted by the Rayleigh criterion. Moreover, the angular resolution of the radar sensor is a system specification that does not only depend on the antenna placement, but also for example on the following signal processing.

The position of the first array, that could either be a transmitter or a receiver, is fixed in the origin of the reference system and the algorithm will find the best position for the $M_{t}+M_{r}-1$ remaining elements.

In this implementation of the genetic algorithm, the chromosomes describe the position of the antennas. Since the optimization is focused only on a multistatic scenario, the positions of the last transmitter and the last receiver must satisfy the condition

$$
x_{T M_{t}}+x_{R M_{r}}=d_{v} .
$$

Afterward, the fitness function must be evaluated for each of the chromosomes in the population. Our definition of the fitness function is closely related to the specific application and in particular it exploits the properties of the AF. A threshold on the value of the AF is fixed and the algorithm in the end maximizes the achievable ambiguity-free region, in which the value of the AF is better than the given threshold.

\section{B. Antenna Array Design}

The antenna system is composed of five arrays, two transmitters and three receivers. Each array is an 8-element cavity antenna array based on the design presented in [10]. As shown in Fig. 2, the antenna array is designed as a twolayer structure. Both the bottom and the top layers employ the substrate RO3003 with nominal permittivity $\varepsilon_{r}=3$ from Rogers Corporation. The upper layer has a thickness of $254 \mu \mathrm{m}$ and the lower one of $127 \mu \mathrm{m}$. Between the top and the bottom layer a bonding film is placed, namely RO3001.

Unlike the design described in [10], in this case a series feeding is employed. The series-fed design needs a smaller area occupation compared to the corporate one and it gives therefore more degrees of freedom for the placement of the antenna arrays on the PCB.

The genetic algorithm described in the previous section has been used to find the correct antenna placement. As additional constraint, the minimum distance between the antenna arrays must be larger than $1.17 \lambda_{0}$ at $77 \mathrm{GHz}$ to avoid overlapping of the antenna columns. The final positions for the arrays are

$$
\boldsymbol{x}_{t}=\left[0,2.54 \lambda_{0}\right], \boldsymbol{x}_{r}=\left[10.81 \lambda_{0}, 12.69 \lambda_{0}, 14.13 \lambda_{0}\right],
$$




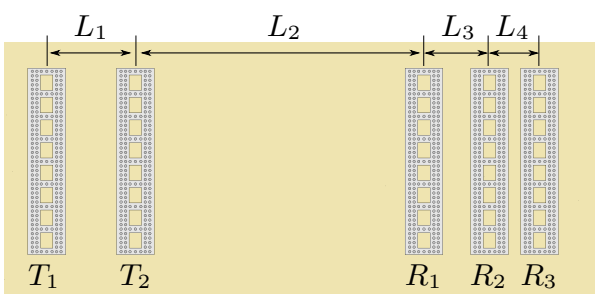

Fig. 3. Schematic of the MIMO configuration. The distance between the antenna arrays are the following (in $\mathrm{mm}$ ): $L_{1}=9.9, L_{2}=32.2, L_{3}=7.3$, and $L_{4}=5.6$. $T_{i}$ and $R_{j}$ describe the transmitters and receivers, respectively.

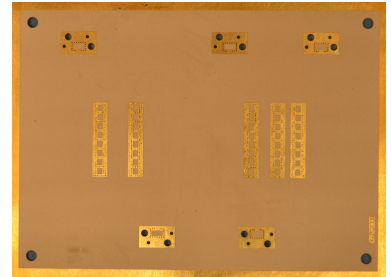

(a)

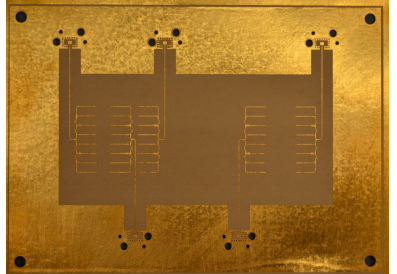

(b)
Fig. 4. Fabricated prototype: (a) front view and (b) back view.

where $\boldsymbol{x}_{t}$ and $\boldsymbol{x}_{r}$ are the vectors containing the positions of the arrays in terms of free-space wavelength at $77 \mathrm{GHz}$. This arrangement of the arrays sets a virtual aperture of $d_{v}=16.67 \lambda_{0}$, which corresponds to an ideal value of angular resolution of $4.2^{\circ}$ according to (4). A schematic of the antennas configuration is depicted in Fig. 3.

\section{EXPERIMENTAL CHARACTERIZATION}

The fabricated prototype can be seen in Fig. 4. Similarly to what is described in [10], due to measurement purposes the microstrip feeding networks are connected to a transition from microstrip line to rectangular waveguide (WR-12). The transitions are integrated in a single metal frame that gives mechanical stability to avoid any bending of the structure during the measurements. The total dimensions of the board are $9.9 \mathrm{~cm} \times 7.1 \mathrm{~cm}$.

\section{A. Measurement Setup}

The two-way radiation pattern has been measured in an anechoic chamber. As a target, a corner reflector with a side length of $6.1 \mathrm{~cm}$ is placed at a distance of approximately $2.2 \mathrm{~m}$.

As can be seen from Fig. 5, two frequency converter modules are connected to a transmitting and receiving array. The frequency converters extend the available frequency range of the vector network analyzer (VNA) up to the E-band (60$90 \mathrm{GHz}$ ). The frequency converters, and thus the PCB too, are placed on a turntable movable in azimuth direction. To increase the dynamic range of the VNA, the resolution bandwidth has been reduced to $500 \mathrm{~Hz}$.

The coupling between the closest antenna array pair has been measured and it is smaller than $-30 \mathrm{~dB}$ in the frequency range between $70 \mathrm{GHz}$ and $85 \mathrm{GHz}$. Since the maximum gain of each antenna array is approximately $13 \mathrm{~dB}$ and due to the free-space attenuation, the amplitude of the collected signal

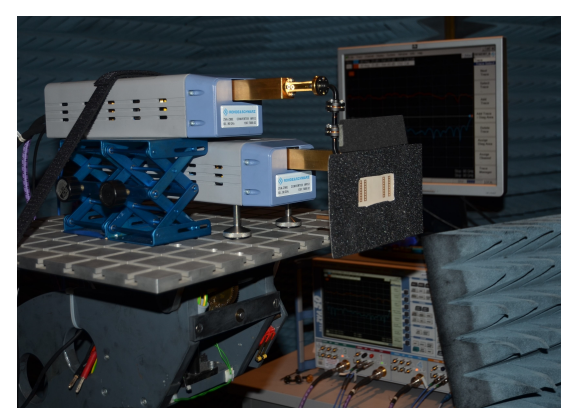

Fig. 5. Measurement setup for the estimation of the two-way pattern.

is smaller than the mutual coupling between a transmitterreceiver pair. Hence, to collect the signal of interest, i.e. the signal backscattered by the target, the time gating must be applied.

Before collecting the measurements, a TRL calibration has been performed that sets the reference plane at the output of the frequency converter modules. To maximize the resolution response of the time gating method, a frequency span of $30 \mathrm{GHz}$ has been used, which is the largest possible with this measurement setup and covers the complete E-band.

\section{B. Measurement Results}

First of all, the reflection coefficient of all the transmitting and receiving arrays has been measured. All the arrays show a reflection coefficient equal to or lower than $-10 \mathrm{~dB}$ in the frequency range of interest $(76 \mathrm{GHz}-81 \mathrm{GHz})$.

Afterward, the two-way radiation pattern for all transmitterreceiver pairs has been measured. While a pair of arrays is connected to the frequency converters, the remaining three arrays in the configuration are matched by waveguide terminations.

As can be seen from Fig. 5, the transmitting array is connected directly to one frequency converter, while to connect the receiving one to the output port of the converter module some waveguide bends and sections are needed. Their presence will affect the phase of the measured two-way pattern and to a smaller extent the amplitude, too. Although not presented in this work, the effect of these waveguide components could be easily compensated in the measurement results.

Figure 7 shows the measured relative amplitude of the twoway radiation pattern at $77 \mathrm{GHz}$ for all the transmitter-receiver pairs. Similar results are obtained for the other frequency points in the frequency range of interest but they are here omitted to avoid duplications. The measured pattern is not perfectly smooth. Indeed, cavity antennas suffer from strong surface wave excitations that may cause ripples in the radiation pattern [10].

Using the measurement results of the two-way pattern, the $\mathrm{AF}$ has been evaluated and it is plotted in Fig. 6a. As can be seen from the picture, some spots with a high value of correlation are apparent; they are due to the ripples measured in the two-way pattern. By considering a threshold of 0.6 , the ambiguity-free region is confined within $\pm 15^{\circ}$. As a comparison, the AF for the ideal case of isotropic radiators is depicted in Fig. 6b. In the ideal case, obviously, there are no high-correlation spots out of the main diagonal, but as can 


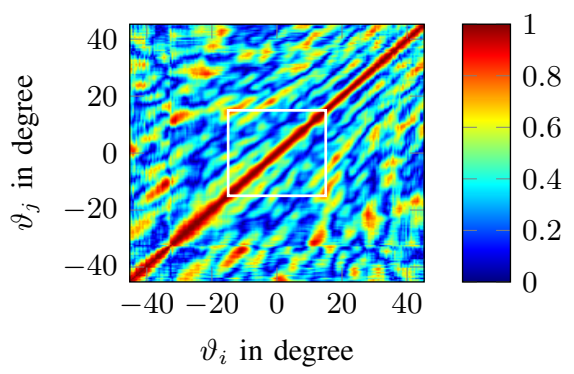

(a)

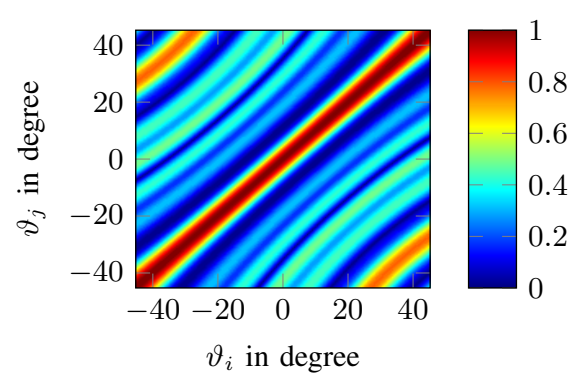

(b)

Fig. 6. (a) AF generated by using the measured two-way patterns at $77 \mathrm{GHz}$; the square shows the ambiguity-free region. (b): AF for isotropic radiators.
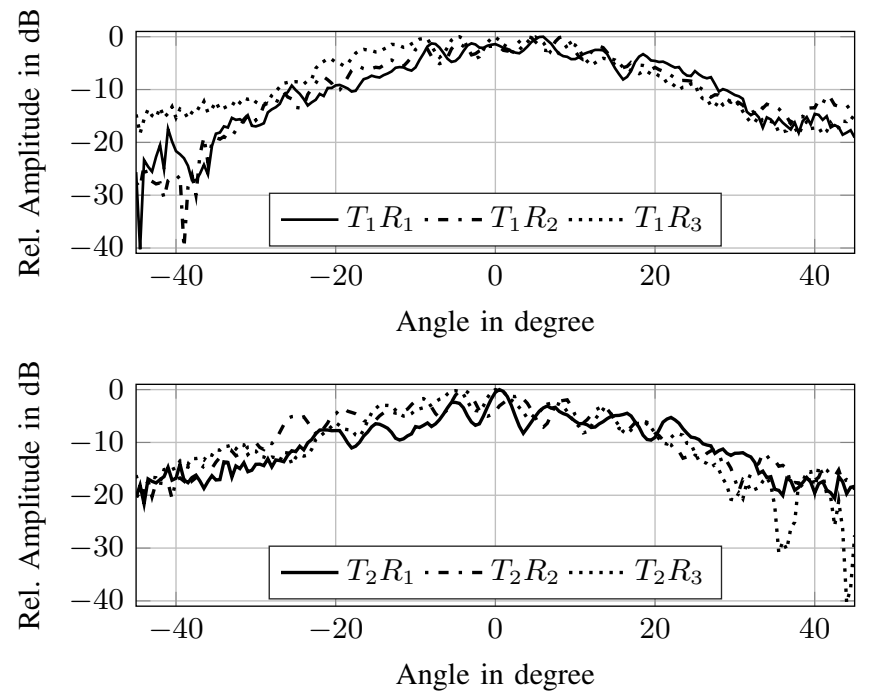

Fig. 7. Measured two-way radiation pattern at $77 \mathrm{GHz}$ using (top) the transmitter $T_{1}$ and (bottom) $T_{2}$.

be seen from the larger width of the main diagonal, the angular resolution is worse than in any practical case.

Although the ambiguity-free region is bounded between $\pm 15^{\circ}$, for the specific automotive application it is also important to check if some ambiguities or near-ambiguities fall inside the FoV of the sensor. In Fig. 6a a high correlation spot is visible, e.g. at $\left(\vartheta_{i}=18^{\circ}, \vartheta_{j}=0^{\circ}\right)$. If this high ambiguity point fall inside the FoV, the performance of the sensor could be severely degraded. For example, an object on the side of the street, i.e. the guardrail, might be detected as an obstacle causing a wrong emergency braking intervention. Hence, only a joint analysis of the ambiguity function and of the FoV can fully describe the performances of an automotive radar sensor.

The antenna arrays have been investigated by means of full-wave simulations. Since it is not possible to simulate the complete antenna system depicted in Fig. 3 due to the complexity of this model, only one transmitter-receiver pair has been simulated and it can be assumed that similar results are obtained for all the pairs. It is unnecessary to use the fullwave simulation results for the calculation of the AF, since the same results as the isotropic case are expected. Thus, the comparison between the ideal isotropic case and measurements is fair for this work.

As final remark, it is important to notice that during the measurement of the two-way pattern it is not possible to turn both the transmitter and receiver at the same time around their own phase center. Thus, this leads to an unavoidable phase error in the measured complex radiation pattern. However, this error can be viewed as a measurement artifact and no additional phase error is expected when the antenna system will be integrated in the radar sensor.

\section{CONCLUSION}

This paper gives an accurate description of the characterization of a mm-wave antenna configuration for MIMO radar applications. The proposed measurement setup allows the assessment of two important metrics for a MIMO radar, the two-way radiation pattern and the ambiguity function, that describes the resolution properties of the antenna system. To confirm the feasibility of the proposed approach, a MIMO antenna configuration based on cavity antenna arrays has been designed and successfully tested.

\section{REFERENCES}

[1] J. Hasch, E. Topak, R. Schnabel, T. Zwick, R. Weigel, and C. Waldschmidt, A Millimeter-Wave Technology for Automotive Radar Sensors in the $77 \mathrm{GHz}$ Frequency Band, IEEE Trans. Microw. Theory Tech., vol. 60, no. 3, pp. 845-860, 2012.

[2] C. Waldschmidt and $\mathrm{H}$. Meinel, Future trends and directions in radar concerning the application for autonomous driving, 44th European Microw. Conf. (EuMC), Rome, 2014, pp. 1719-1722.

[3] S. Lutz, T. Walter, and R. Weigel, Lens-based $77 \mathrm{GHz}$ MIMO radar for angular estimation in multitarget environments, Int. Journal of Microw. and Wireless Tech., vol. 6, pp. 397-404, 2014.

[4] J. Li and P. Stoica, MIMO Radar with Colocated Antennas, in IEEE Signal Process. Mag., vol. 24, no. 5, pp. 106-114, Sept. 2007.

[5] H. Chen, X. Li and Z. Zhuang, Antenna Geometry Conditions for MIMO Radar With Uncoupled Direction Estimation, in IEEE Trans. Antennas Propag., vol. 60, no. 7, pp. 3455-3465, July 2012.

[6] M. Eric, A. Zejak, and M. Obradovic, Ambiguity characterization of arbitrary antenna array: type I ambiguity, Proc. IEEE 5th Int. Symp. on Spread Spectrum Tech. and Appl., Sun City, 1998, pp. 399-403, vol. 2.

[7] J. Salmi and A. F. Molisch, Propagation Parameter Estimation, Modeling and Measurements for Ultrawideband MIMO Radar, in IEEE Trans. Antennas Propag., vol. 59, no. 11, pp. 4257-4267, Nov. 2011.

[8] A. A. H. Azremi, M. Costa, V. Koivunen, and P. Vainikainen, Ambiguity analysis of isolation - based multi-antenna structures on mobile terminal, Proc. 5th European Conf. Antennas Propag. (EuCAP), Rome, 2011, pp. 552-556.

[9] R. L. Haupt, An Introduction to Genetic Algorithms for Electromagnetics, in IEEE Antennas Propag. Mag., vol. 37, no. 2, pp. 7-15, Apr. 1995.

[10] C. Vasanelli, T. Ruess, and C. Waldschmidt, A 77- GHz Cavity Antenna Array in PCB Technology, IEEE 15th Mediterranean Microw. Symp. (MMS), 2015, Lecce, pp. 1-4. 